\title{
从烯烃与碳酸铵无金属催化氢胺化一步法合成伯胺
}

\author{
于 涛 李鹏飞* \\ (西安交通大学前沿科学技术研究院 西安 710054)
}

\section{One-Step Access to Primary Amines from Alkenes and Ammonium Carbonate by One-Step Metal-Free Catalysis}

\author{
Yu, Tao \\ Li, Pengfei* \\ (Frontier Institute of Science and Technology, Xi'an Jiaotong University, Xi'an 710054)
}

脂肪伯胺是一类广泛存在于药物分子、纺织材料、 农药等中的重要结构, 且由于其结构特性(亲水性与疏 水性并存), 脂肪伯胺拥有优秀的表面性能(Scheme 1). 除此之外, $\mathrm{N}$ 原子上的活泼氢能够与多种官能团相互转 化, 这又使伯胺在化学、化工和材料科学中具有极其重 要的地位 ${ }^{[1]}$. 但基于脂肪伯胺的高反应活性, 研究者在 设计选择性合成的过程中往往会面临巨大的挑战. 目前 合成脂肪伯胺的主要方法包括: 过量氨对烷基卤化物的 $\mathrm{S}_{\mathrm{N}} 2$ 取代 ${ }^{[2]} 、$ Gabriel 反应 ${ }^{[3]} 、$ Staudinger 反应 ${ }^{[4]}$ 以及对 烷基硝基、腈、肜或亚胺的还原反应. 虽然其中一些方 法已被应用于工业生产, 但在实际操作中依然不乏局限 性: (1)由于伯胺的高反应活性, 反应产物往往难于停在 伯胺产物, 通常伴随仲胺、叔胺产物一起生成; (2)只能 合成线性或 $\alpha$-支链的伯胺, 难以合成位阻更大的 $\alpha$-叔碳 伯胺; (3)反应步骤繁琐, 条件苛刻, 且原料昂贵或有毒. 鉴于此, 金属催化的烯烃氢胺化反应遂成为近年来科学 家们研究的热点 ${ }^{[5]}$.

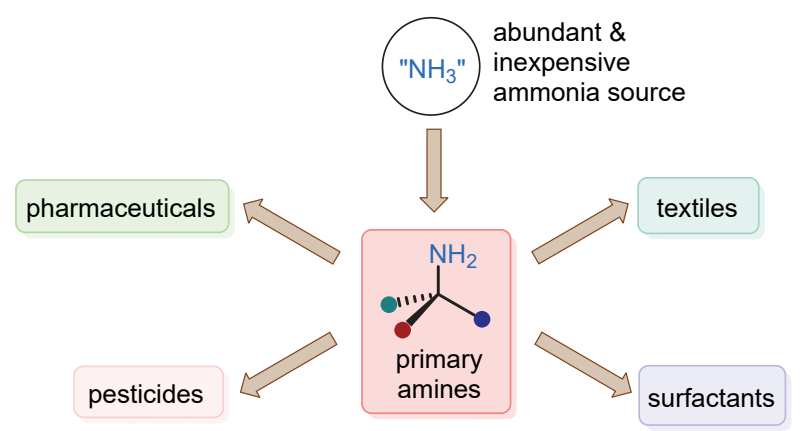

图式 1 合成脂肪伯胺的重要性

Scheme 1 Significance of synthetic aliphatic primary amines
作为石油化工中易得的化工原料, 烯烃以其提供获 得分子复杂性和后期官能团化的特性, 被应用于氢胺化 反应已达数十年之久. 但就未活化的烯烃与胺源分子间 偶联生成脂肪族伯胺的方法而言, 目前尚不多见 ${ }^{[6]}$. 2018 年, Buchwald 课题组 ${ }^{[7]}$ 报道了以苯并异噁唑亲电试 剂为潜在胺源, 用铜催化烯烃氢胺化经两步合成伯胺的 方法. 遗憾的是, 由于三取代和四取代的烯烃空间位阻 所导致的烯烃与金属中心的亲和力较低, 最终难以获得 $\alpha$-叔伯胺产物. 随后, Knowles 课题组 ${ }^{[8]}$ 通过将伯胺或仲 胺的单电子氧化生成的铵基阳离子中间体与烯烃偶联, 制备了一系列仲胺和叔胺. 然而这一方法并不适用于伯 胺的直接合成.

近日，南方科技大学化学系舒伟课题组 ${ }^{[9]}$ 采用简 单、廉价、易得、传统上用于化肥的碳酸铵作为氨源, 以 一个有机盐 $\mathrm{Mes}-\mathrm{Acr}-\mathrm{Ph}^{+}\left(E_{\text {red }}^{*}=+2.20 \mathrm{~V}\right)$ 作为光催化 剂, 实现了从烯烃直接获得伯胺的氢胺化反应. 作者通 过对广泛的底物种类进行测试, 证明了用上述方法可被 用于药物和天然产物合成中高度功能化的脂肪族伯胺 的实际合成(Scheme 2).

该方法以烯烃和碳酸铵为原料, 在温和的反应条件 下即可直接制备脂肪伯胺化合物. 值得一提的是, 反应 中氨源的释放速率对反应具有显著影响. 碳酸铵作为氨 源是必要的, 其次 2-氨基苯硫酚中的氨基能够与硫醇相 互作用促进氢原子转移, 从而大大提升反应活性. 在优 化条件下，作者考察了各种类型烯烃，包括末端烯烃、 非末端烯烃、芳基烯烃、烷基烯烃、烯基醚及烯基酰胺 等. 含有多种官能团的苯乙烯类底物均可以 $55 \% \sim 99 \%$ 的产率得到相应的脂肪族伯胺产物; 对于难以合成的 $\alpha$ -

\footnotetext{
* Corresponding author. E-mail: lipengfei@mail.xjtu.edu.cn. Published online June 16, 2021.
} 
叔碳脂肪族伯胺也能以 $52 \% \sim 79 \%$ 的产率实现. 该反应 可以合成 1,2-氨基醇、1,2-二胺、2-氨基醚等衍生物; 也 适用于多种含烯烃片段的天然产物衍生物, 从而高效地 构建相应的伯胺化合物. 值得一提的是, 基于不同类型 烯烃反应性的差异，该反应可实现同一原料中多取代烯 烃的选择性氢胺化反应.

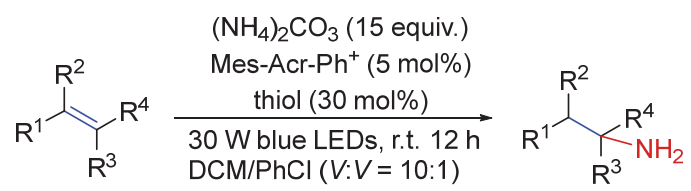<smiles>Cc1cc(C)c(-c2c3ccccc3nc3ccccc23)c(C)c1</smiles>

$\begin{array}{ll}\mathrm{Ph} & \mathrm{BF}_{4}^{-}\end{array}$

Mes-Acr-Ph ${ }^{+}$

Selected examples:<smiles>Cc1ccc(C)c(OCC(=O)OCc2ccccc2)c1</smiles>

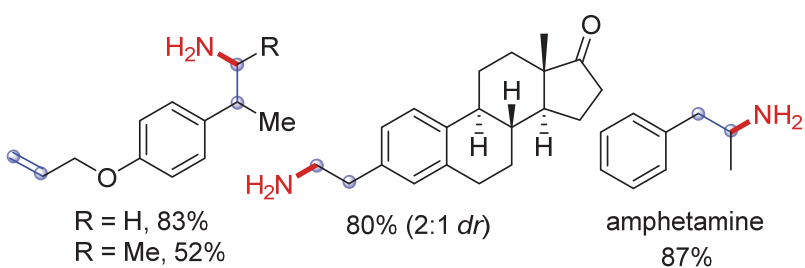

图式 2 无金属催化的烯烃氢胺化反应

Scheme 2 Selective metal-free hydroamination

作者对反应机理进行了一系列研究, 认为该反应很 可能经过烯烃部分的单电子氧化得到自由基阳离子中 间体, 然后与碳酸铵提供的氨源反应, 得到目标氢胺化 产物(Scheme 3). 反应中的光催化剂(PC)经可见光激发 形成激发态 $\mathrm{PC}^{*}$, 通过单电子氧化与烯烃相互作用, 形 成还原物种 PC-1, 烯烃则生成自由基阳离子中间体 M1. M1 与碳酸铵释放出的氨结合, 形成中间体 M2. M2 的 自由基稳定性决定了氨进攻的区域选择性. M2 可以与
2-氨基苯硫酚发生氢原子转移(HAT), 构成质子化伯胺 M3 和中间体 M4. PC-1 可还原 M4 再生 PC 和 2-氨基苯 硫酚负离子 M5, M3 可将其质子化生成 2-氨基苯硫酚和 伯胺产物.

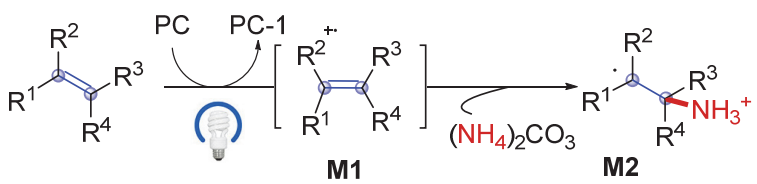

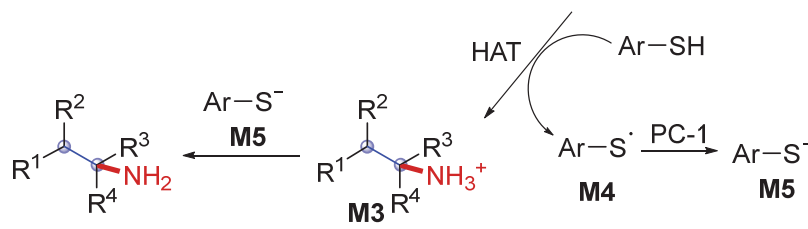

图式 3 烯烃氢胺化反应机理

Scheme 3 Mechanism of the alkene hydroamination reaction

总之, 舒伟课题组发展了可见光促进下基于烯烃自 由基阳离子中间体烯烃与碳酸铵的氢胺化反应. 该反应 为药物和天然产物的合成、衍生中制备各种脂肪伯胺类 化合物提供了一种通用的、直接的合成方法.

\section{References}

[1] Lawrence, S. A. Amines: Synthesis Properties and Applications, Cambridge University Press, Cambridge, 2004.

[2] Wade, L. G., Jr.; Simek, Jr., J. W. Organic Chemistry, 9th ed., Pearson, San Fran-cisco, 2017, 1020.

[3] Gibson, M. S.; Bradshaw, R. W. Angew. Chem., Int. Ed. Engl. 1968, 7, 919; Angew. Chem. 1968, 80, 986.

[4] "Staudinger Reaction": Kürti, L.; Czakó, B. in Strategic Applications of Named Reactions in Organic Synthesis, 1st ed., Academic Press, New York, 2005, p. 428.

[5] Bronner, S. M.; Grubbs, R. H. Chem. Sci. 2014, 5, 101.

[6] Howk, B. W.; Little, E. L.; Scott, S. L.; Whitman, G. M. J. Am. Chem. Soc. 1954, 76, 1899

[7] Guo, S.; Yang, J. C.; Buchwald, S. L. J. Am. Chem. Soc. 2018, 140, 15976.

[8] (a) Musacchio, A. J.; Lainhart, B. C.; Zhang, X.; Naguib, S. G.; Sherwood, T. C.; Knowles, R. R. Science 2017, 355, 727.

(b) Miller, D. C.; Ganley, J. M.; Musacchio, A. J.; Sherwood, T. C.; Ewing, W. R.; Knowles, R. R. J. Am. Chem. Soc. 2019, 141, 16590.

[9] Du, Y.-D.; Chen, B.-H.; Shu, W. Angew. Chem., Int. Ed. 2021, 60, 9875. 